\title{
Secondary choroidal neovascularization due to choroidal osteoma after 9 years follow-up
}

\author{
Yun Zhang ${ }^{1}$, Jia Fang ${ }^{2}$, Shixin Zhao ${ }^{1}$, Xiangjun She ${ }^{1}$, Jun Wang ${ }^{1}$ and Lijun Shen ${ }^{1 *}$ (1)
}

\begin{abstract}
Background: Choroidal osteoma is a benign intraocular tumor that can increase risk of developing choroidal neovascularization. The visual prognosis is influenced by the tumor location, decalcification status, overlying RPE atrophy, presence of choroidal neovascularization, persistence of subretinal fluid and occurrence of subretinal hemorrhages.
\end{abstract}

Case presentation: The authors present a 40-year-old woman diagnosed with choroidal osteoma of the right eye. Her best corrected visual acuity was 12/20 but decreased to 5/20 due to secondary choroidal neovascularization after 8 years follow up. Fundus examination revealed an enlarged choroidal osteoma in most margins at posterior pole with schistose hemorrhage beside macula. Optical coherence tomography angiography revealed unique features in the vascular changes of choroidal neovascularization in choroidal osteoma in the outer retinal layer and choroid capillary layers, and subretinal neovascularization. Indocyanine green fluorescence angiography showed there was hypo-fluorescence at the peripapillary with faint hyper-fluorescence at the macular, corresponding to the location on the fundus photograph. The patient received 3 injections of intravitreal ranibizumab. After 1 year follow up, her visual acuity of the right eye was 18/20 and the CNV had regressed.

Conclusions: We present the findings and treatment of a case of choroidal osteoma with secondary choroidal neovascularization. Optical coherence tomography angiography combined with FFA and ICGA is used to analysis the characteristics of secondary choroidal neovascularization. Optical coherence tomography angiography can reveal some unique characteristics in the vascular changes compared to fundus fluorescein angiography.

Keywords: Choroidal osteoma, Secondary choroidal neovascularization, 9 years follow-up

\section{Background}

Choroidal osteoma(CO) is a rare, benign, ossifying tumor within the choroid of unknown etiology [1]. CO is always found in young healthy females about 20 or 30 years old, with no history of systemic or ocular disease. $80 \%$ of cases is unilateral clinically and tends to occur choroidal neovascularization $(\mathrm{CNV})$ in up to one-third of cases [2]. The initial descriptions of choroidal osteoma

\footnotetext{
* Correspondence: slj@mail.eye.ac.cn

${ }^{1}$ Affiliated Eye Hospital of Wenzhou Medical University, Hangzhou, Zhejiang, China

Full list of author information is available at the end of the article
}

was by Gass in 1978 [3], as a slightly elevated, juxtapapillary, yellowish orange, choroidal lesion with well-defined margins. Fundus fluorescein angiography (FFA), indocyanine green fluorescence angiography (ICGA) and optical coherence tomography angiography (OCTA) as ancillary diagnostic tests may help demonstrate choroidal osteoma and detect RPE atrophy, CNV formation and the characteristic spider vessels, which may be amenable to treatment by directing for complications arising from $\mathrm{CNV}$ and subretinal fluid.

(c) The Author(s). 2021 Open Access This article is licensed under a Creative Commons Attribution 4.0 International License, which permits use, sharing, adaptation, distribution and reproduction in any medium or format, as long as you give appropriate credit to the original author(s) and the source, provide a link to the Creative Commons licence, and indicate if changes were made. The images or other third party material in this article are included in the article's Creative Commons licence, unless indicated otherwise in a credit line to the material. If material is not included in the article's Creative Commons licence and your intended use is not permitted by statutory regulation or exceeds the permitted use, you will need to obtain permission directly from the copyright holder. To view a copy of this licence, visit http://creativecommons.org/licenses/by/4.0/ The Creative Commons Public Domain Dedication waiver (http://creativecommons.org/publicdomain/zero/1.0/) applies to the data made available in this article, unless otherwise stated in a credit line to the data. 
In this case report we using multimodal imaging to describe the change of this disease after 9 years follow-up, especially the secondary choroidal neovascularization.

\section{Case presentation}

A 40-year-old woman who first presented with complaints of decreased vision and metamorphopsia in her right eye was diagnosed with $\mathrm{CO} 9$ years ago. At that time her best corrected visual acuity (BCVA) was $12 / 20$. Both eyes had normal anterior segments. Right fundus examination showed a geographic-shaped, yellowishwhite choroidal lesion surrounding the optic disc in the right eye (Fig. 1). B-scan ultrasonography of right eye revealed a typical dense echogenic plaque which causing acoustic shadowing behind (Fig. 2). FFA and ICGA had no evidence of CNV except early hyperfluorescent choroidal filling pattern with late diffuse staining. Computerized tomography (CT) showed a hyperdense choroidal plaque with the same density as bone (Fig. 3). Optical coherence tomography (OCT) demonstrated serous retinal detachments at the initial examination (Fig. 4).

There was no evidence of any subretinal neovascularization clinically and the patient maintained stable vision, so she was asked to come for regular follow-up.

Last year, after 8 years follow up, secondary CNV was found by angio-OCT at the temporal of CO. BCVA had decreased to 5/20. B-scan and CT didn't demonstrate much different than before. Compared to 8 years ago, $\mathrm{CO}$ grew up in most margins and macular appeared schistose hemorrhage (Fig. 5). A part of tumor on the inferior margin displayed decalcification and choroid atrophy. OCT revealed subretinal neovascularization with choriocapillaris atrophy. OCTA revealed superficial and deep subretinal neovascularization of $\mathrm{CNV}$ in $\mathrm{CO}$ not

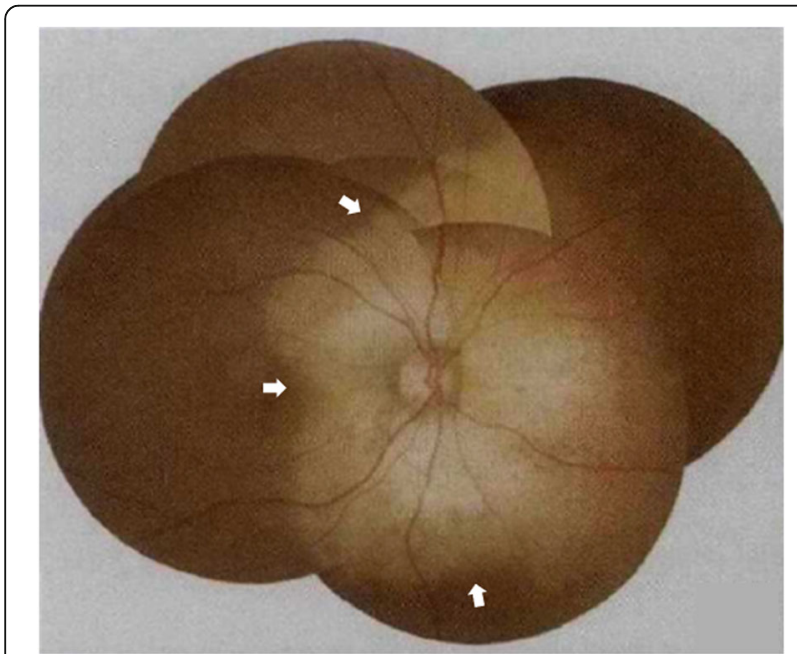

Fig. 1 Fundus photograph showed a yellowish white, peripapillary and sharply demarcated choroidal lesion involving the macula (white arrows)

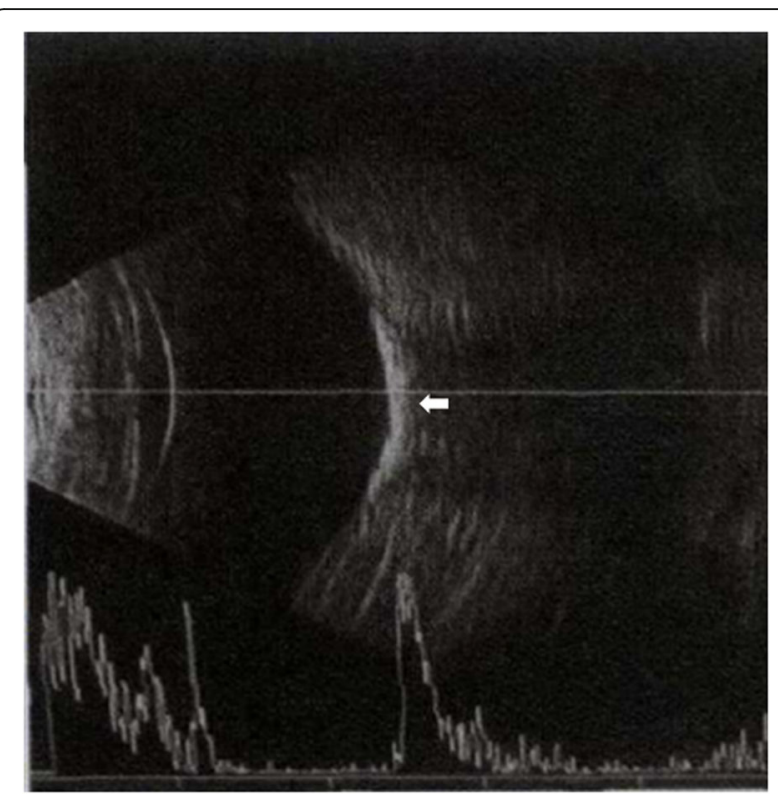

Fig. 2 B-scan demonstrated focal subretinal calcification next to the optic disc (white arrow)

visualize with other imaging methods (Fig. 6). Except CO, ICGA didn't show the morphology and structure of secondary choroidal neovascularization as obvious as OCTA. (Fig. 7).

Based on those findings, the patient was diagnosed with secondary CNV. The patient was administered 3 intravitreal ranibizumab injections at 1 -month intervals. In follow-up examination at 3 months post-injections, visual acuity had improved to $18 / 20$ and OCT showed regression of the subretinal fluid (Fig. 8). The patient's

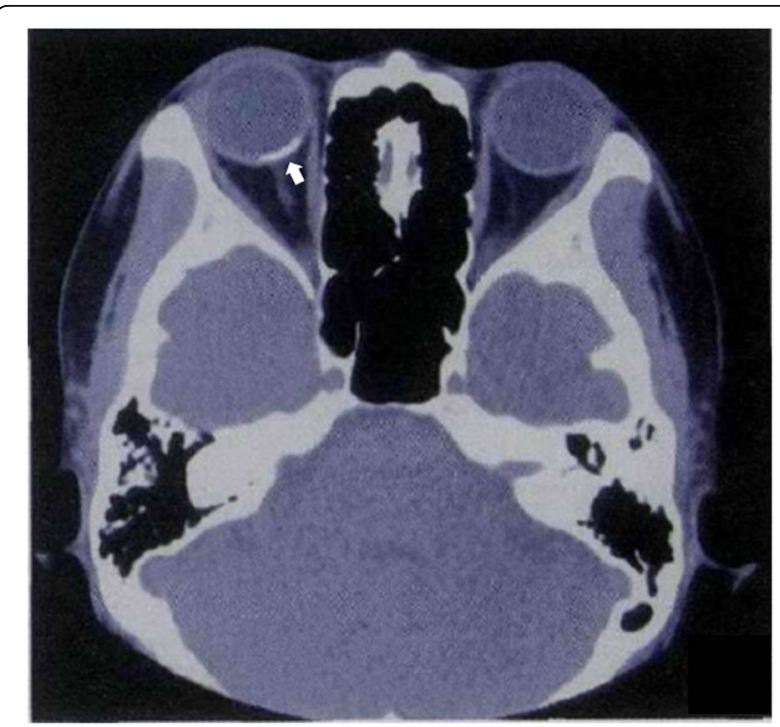

Fig. $3 \mathrm{CT}$ demonstrated a hyperdense choroidal plaque with the same density as bone typically (white arrow) 

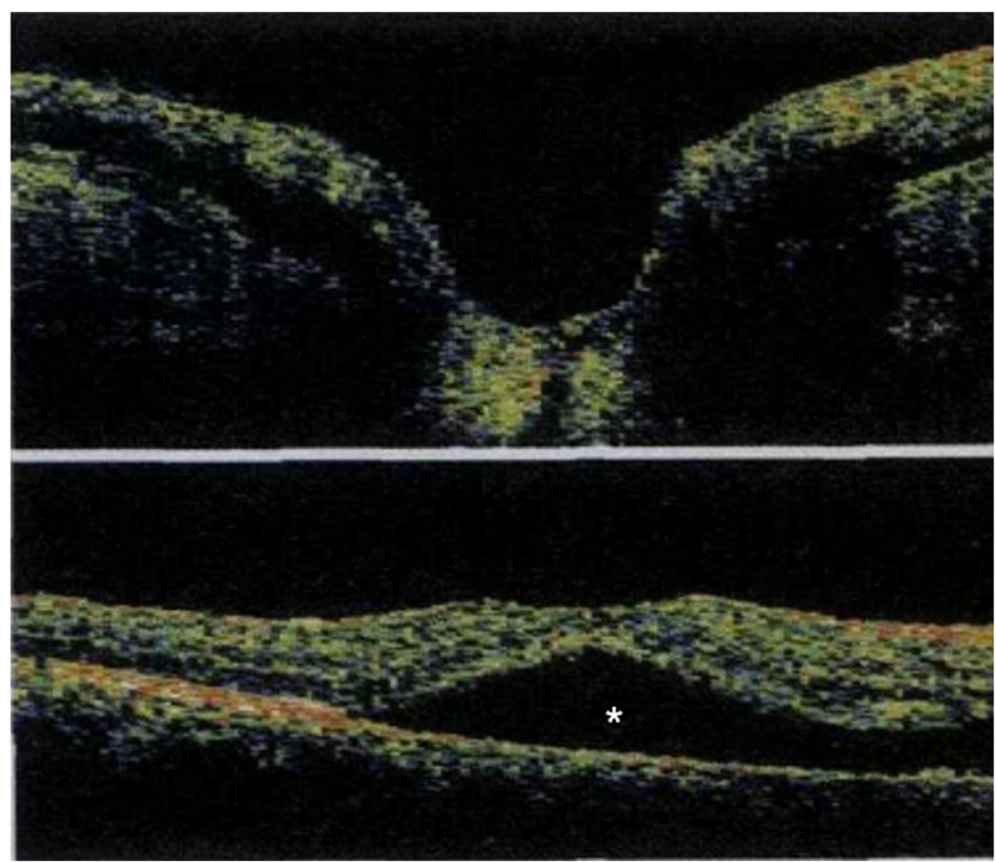

Fig. 4 OCT showed serous fluid exudates under the macula (white asterisk)

condition was stable during the 1-year follow-up period and no additional injections were required. OCTA also showed reduction in area of CNV (Fig. 9).

\section{Discussion and conclusion}

This case report illustrates the need for prolonged follow-up. The importance of long-term follow-up is even more central to the full assessment and treatment

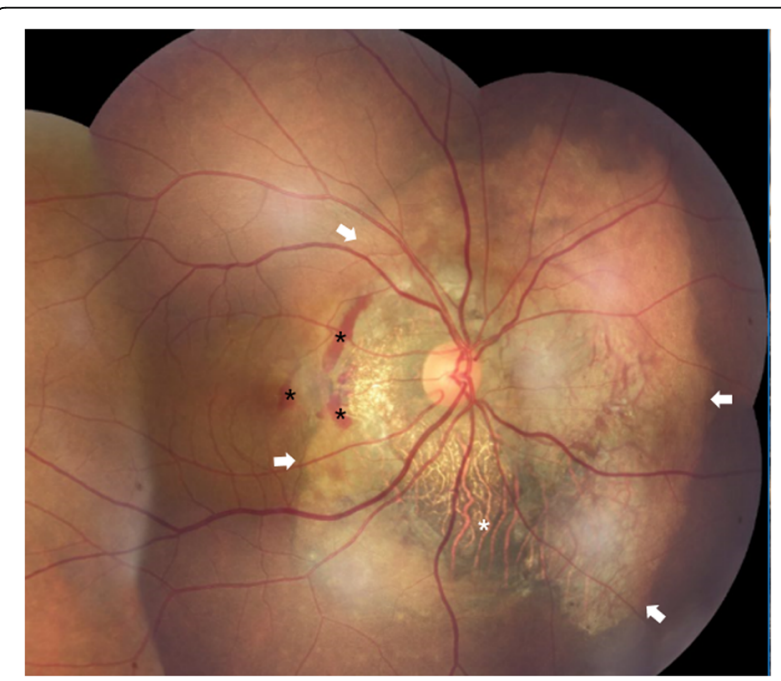

Fig. 5 Right fundus showed an enlarged choroidal osteoma in most margins at posterior pole (white arrow) with schistose hemorrhage (black asterisk) beside macula compared to eight years ago. Partial decalcification on the inferior margin and visibility of large choroidal vessels were noted (white asterisk) in malignant tumors. Patients with benign tumors also require long-term surveillance to monitor morbidities of intraocular complications. The most important complications of $\mathrm{CO}$ are subretinal neovascularization, subretinal and intraretinal hemorrhages, and serous and hemorrhagic retinal detachments. Despite being a benign tumor, the complications may cause serious vision loss, especially $\mathrm{CNV}$, subfoveal fluid, and photoreceptor degeneration [4]. At present, the mechanism by which $\mathrm{CNV}$ develops is primarily considered retinal pigment epithelium disruption allows growth of underlying choroidal new vessels together with thinning of Bruch's membrane and the choriocapillaris, or that osteoma itself has neovascular membrane extensions $[5,6]$.

Here, we report a case of $\mathrm{CO}$ associated with the development of CNV. OCTA showed a dense irregular neovascularization network in the outer retinal layer and choroid capillary layers. Due to serous retinal detachment appearing before $\mathrm{CNV}$, we seem to be considered retinal pigment epithelium disruption tends to be CNV more easily. The presence of subretinal fluid has been found to be predictive of CNV [7]. It is also consistent with the mechanism. A long-term follow-up of choroidal osteoma indicated around 64\% of eyes of $\mathrm{CO}$ with subretinal fluid resolved spontaneously maintained good vision [8]. Although this is only for those without CNV. In our case, visual acuity improved from $5 / 20$ to $18 / 20$ after 3 monthly injections and was preserved at this level throughout a 12month follow-up period. 

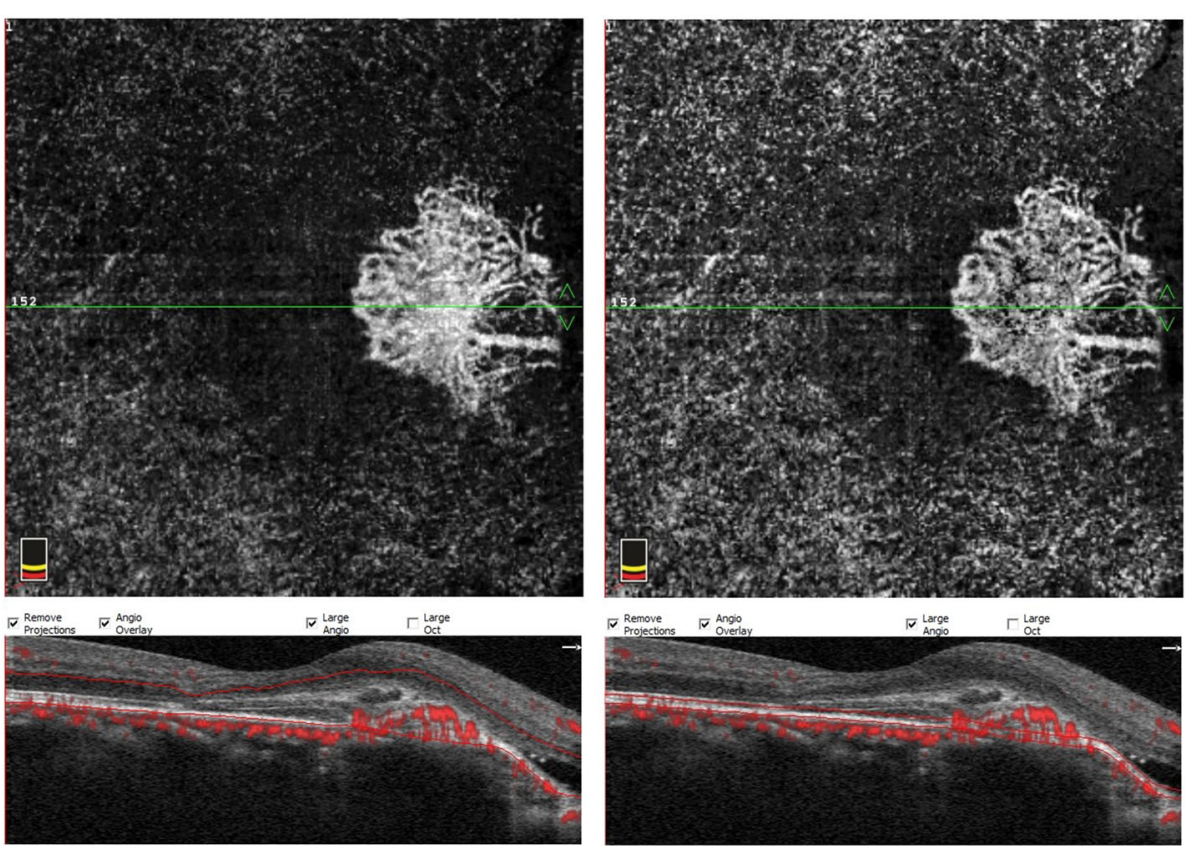

Fig. 6 OCTA revealed unique features in the vascular changes of CNV in CO in the outer retinal layer and choroid capillary layers, and subretinal neovascularization (white arrow)

Some reports also describe tumor growth in $41-64 \%$ of cases followed for a period of 10 years. Most choroidal osteomas have a slow random growth with an increase in mean basal diameter of around $0.37 \mathrm{~mm}$ per year on any of the non-calcified margins [2]. In our case, about $2.96 \mathrm{~mm}$ edge should increase compared to 8 years ago according to the report. However, our case didn't reach

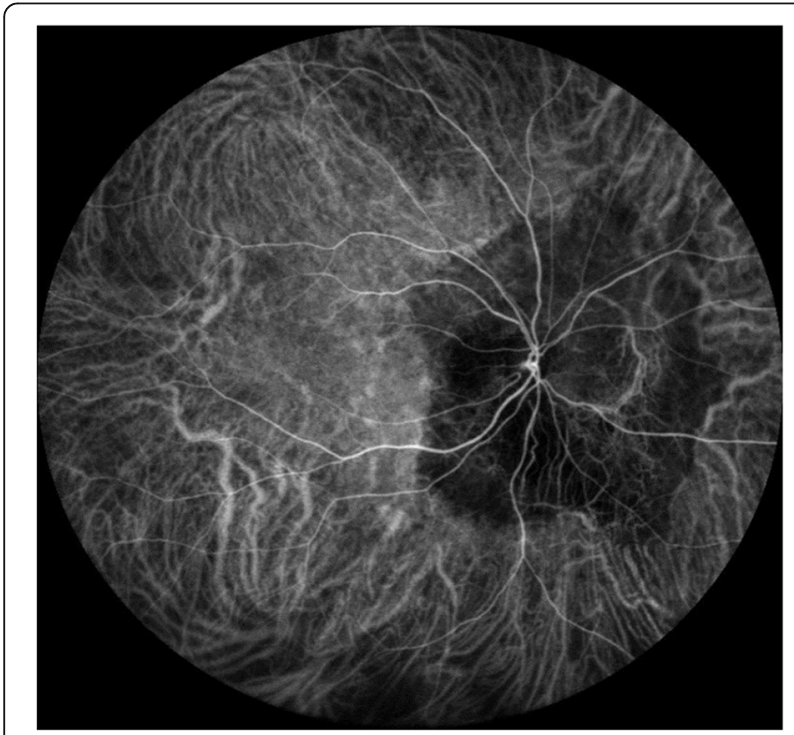

Fig. 7 ICGA showed there was hypo-fluorescence at the peripapillary with faint hyper-fluorescence at the macular, corresponding to the location on the fundus photograph. The subretinal neovascularization of CNV was not obvious the level. On the calcified margins, the growth of $\mathrm{CO}$ almost stopped. The cause of the slower growth may be a result of osteoclastic active in the lesion.

There is no standard of treatment for choroidal osteomas in addition to observing, but therapies are directed for complications arising from $\mathrm{CNV}$ and subretinal fluid. There are some reports describe the clinical and diagnostic features of this tumor, some of them treat related $\mathrm{CNV}$ with photodynamic therapy or/and anti-vascular endothelial growth factor (anti-VEGF) [9, 10]. Some studies showed PDT had achieved success not only in the management of $\mathrm{CNV}$ secondary to choroidal osteoma but also preventing tumor growth toward the foveola $[10,11]$. However, reperfusion following photodynamic therapy might lead to $\mathrm{CNV}$ formation and tumor decalcification $[11,12]$. Furthermore, PDT may require more than once treatment and final visual acuity may decline [13]. In addition, thermal laser photocoagulation and transpupillary thermotherapy may have certain efficacy in cases of CNV secondary to choroidal osteoma while increasing retinal damage $[5,14,15]$.

Another method of controlling CNV growth is intravitreal anti-VEGF. Our case was treated by anti-VEGF and stable for now, in spite of keeping indispensable monitoring. It is reported bevacizumab and ranibizumab have positive outcomes in both anatomy and visual acuity $[9,16]$. The rapid regression of CNV secondary to $\mathrm{CO}$ may attributed to enhanced passage of the ranibizumab through the thinned and degenerated RPE and 

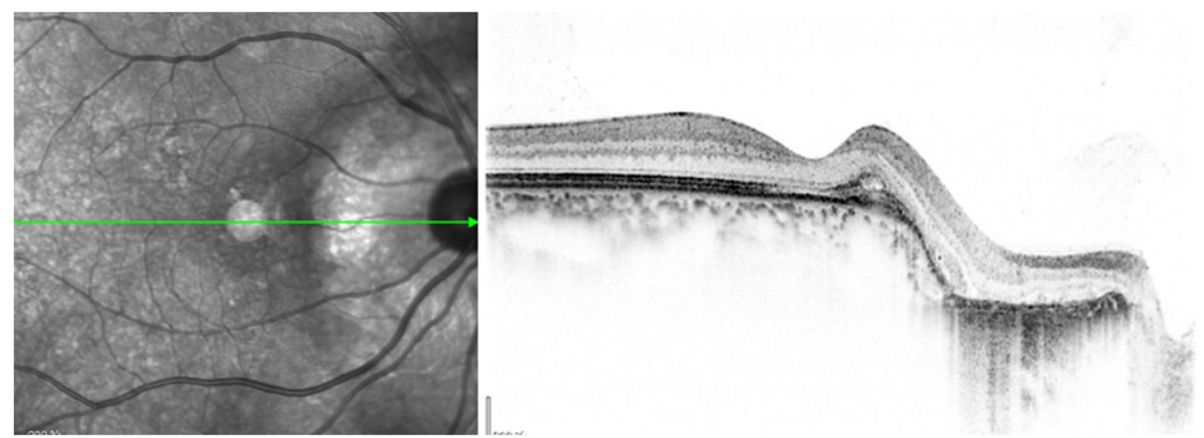

Fig. 8 OCT appreciated CNV and on the nasal atrophic retina

Bruch's membrane to the subretinal area, thus increasing the drug's efficacy [16].

This patient did not show any CNV at the first time, after 8 years the tumor got a little bigger and the secondary CNV was founded. We use angio-OCT combine with FFA and ICGA to analysis the characteristics of these changes. On fluorescein angiography, choroidal osteoma has early patchy hyperfluorescent choroidal filling pattern and late diffuse staining due to leakage surrounded by an area of blocked fluorescence. Fluorescein angiography is also helpful in detecting RPE atrophy, CNV formation or the characteristic spider vessels [2, 17]. However, the leaking vessels of the CNV usually are overshadowed by the dye in the late phases [18]. OCTA as a a novel imaging tool which allows the visualization of the retinal and choroidal vasculature, may demonstrate CNV morphology, vessel parameter, the motion of erythrocytes, and even predict treatment response. A small and rarified vascular network inside a capsular formation was observed of $\mathrm{CNV}$ secondary to $\mathrm{CO}$ [18]. OCTA may reveal some unique characteristics in the vascular changes of $\mathrm{CNV}$ in $\mathrm{CO}$.

To conclude, $\mathrm{CO}$ is benign ossifying tumors of uveal tract. Most choroidal osteomas have a slow random growth, on any of the non-calcified margins. Lifelong clinical follow-up should be maintained to detect complications at early stages. Therapies directed for complications arising from choroidal neovascularization (CNV) are intravitreal anti-VEGF, PDT and laser photocoagulation. OCTA may visualize some micro vascular lesions

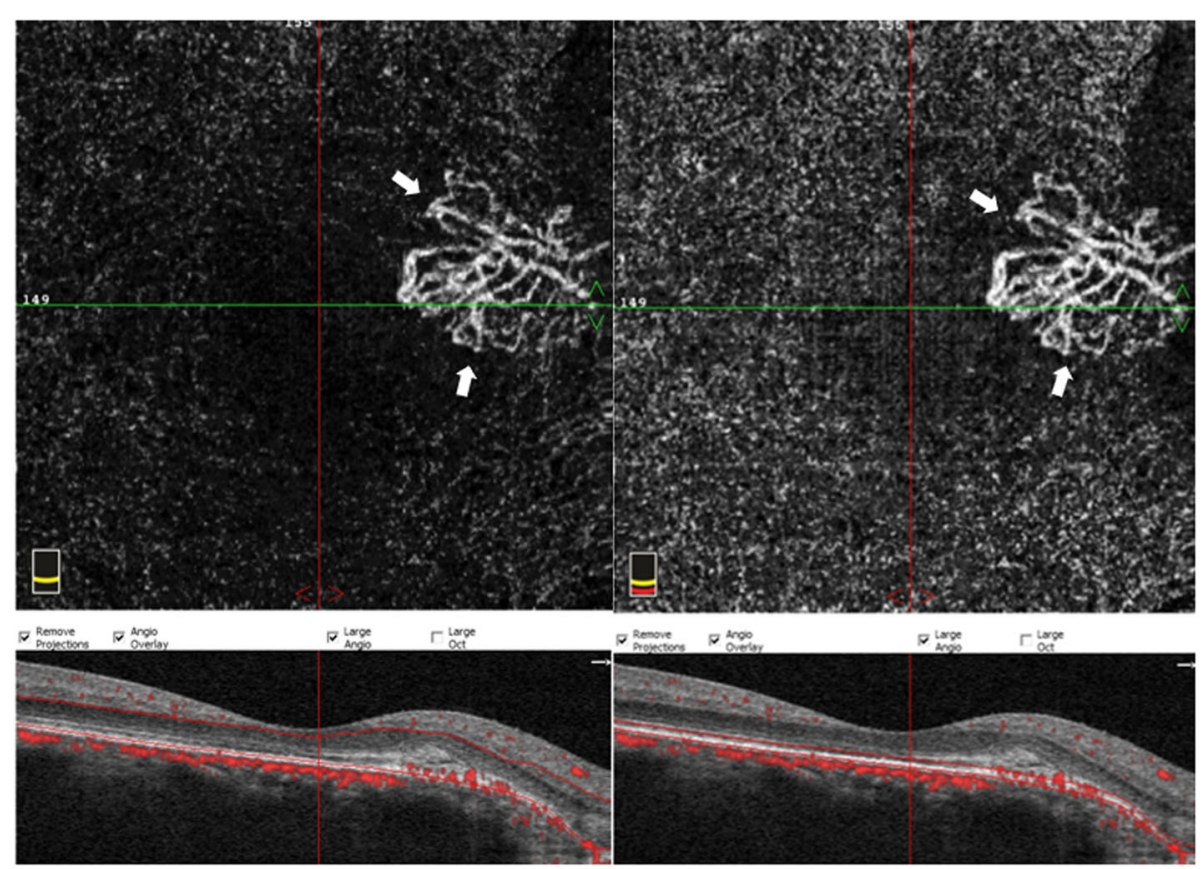

Fig. 9 OCTA revealed the regression of choroidal neovascularization density and range in the outer retinal layer and choroid capillary layers. (white arrow) 
instead of other image methods to some extent, and help diagnosis and follow-up of CNV secondary to $\mathrm{CO}$.

\section{Abbreviations}

CO: Choroidal osteoma; CNV: Choroidal neovascularization; BCVA: Best Corrected Visual Acuity; FFA: Fundus fluorescein angiography; ICGA: Indocyanine green fluorescence angiography; OCTA: Optical coherence tomography angiography; CT: Computerized tomography; OCT: Optical coherence tomography

\section{Acknowledgements}

Not applicable.

\section{Authors' contributions}

YZ conceived and designed the study. JF, SXZ, XJS, JW, LJS and YZ performed the study and analyzed the data at each center. JF and $Y Z$ wrote the manuscript and equally contributed to the manuscript as the first authors. SLJ contributed to the manuscript as the corresponding author. All authors read and approved the final manuscript.

\section{Funding}

No external funding was provided.

\section{Availability of data and materials}

The datasets used and analysed during the current study available from the corresponding author on reasonable request.

\section{Declarations}

\section{Consent for publication}

Informed written consent for the publication of this case and any additional related information was taken from the patients involved in the study.

\section{Competing interests}

The authors declare that they have no competing interests.

\section{Author details}

'Affiliated Eye Hospital of Wenzhou Medical University, Hangzhou, Zhejiang, China. ${ }^{2}$ Yongkang First People's Hospital of Hangzhou Medical College, Jinhua, Zhejiang, China.

Received: 7 January 2021 Accepted: 19 May 202

Published online: 31 May 2021

\section{References}

1. Kadrmas EF, Weiter JJ. Choroidal osteoma. Int Ophthalmol Clin. 1997;37(4): 171-82. https://doi.org/10.1097/00004397-199703740-00015.

2. Alameddine RM, Mansour AM, Kahtani E. Review of choroidal osteomas. Middle East Afr J Ophthalmol. 2014;21(3):244-50. https://doi.org/10.4103/ 0974-9233.134686

3. Gass JD, et al. Choroidal Osteoma. Arch Ophthalmol. 1978;96(3):428-35. https://doi.org/10.1001/archopht.1978.03910050204002

4. Song MH, Roh YJ. Intravitreal ranibizumab in a patient with choroidal neovascularization secondary to choroidal osteoma. Eye (Lond). 2009;23(8): 1745-6. https://doi.org/10.1038/eye.2008.313.

5. Shields CL, Shields JA, Augsburger JJ. Choroidal osteoma. Surv Ophthalmol. 1988:33(1):17-27. https://doi.org/10.1016/0039-6257(88)90069-0.

6. Foster BS, Fernandez-Suntay JP, Dryja TP, Jakobiec FA, D'Amico DJ. Clinicopathologic reports, case reports, and small case series: surgical removal and histopathologic findings of a subfoveal neovascular membrane associated with choroidal osteoma. Arch Ophthalmol. 2003;121(2):273-6. https://doi.org/10.1001/archopht.121.2.273.

7. Shields CL, Sun H, Demirci H, Shields JA. Factors predictive of tumor growth, tumor decalcification, choroidal neovascularization, and visual outcome in 74 eyes with choroidal osteoma. Arch Ophthalmol. 2005; 123(12):1658-66. https://doi.org/10.1001/archopht.123.12.1658.

8. Aylward GW, Chang TS, Pautler SE, Gass JD. A long-term follow-up of choroidal osteoma. Arch Ophthalmol. 1998;1 16(10):1337-41. https://doi. org/10.1001/archopht.116.10.1337.
9. Sarıgül Sezenöz A, Bayar SA, YIImaz G. Choroidal Osteoma and Secondary Choroidal Neovascularization Treated with Ranibizumab. Turk J Ophthalmol. 2017:47(4):243-6. https://doi.org/10.4274/tjo.86658.

10. Mazloumi M, Dalvin LA, Ancona-Lezama D, Mashayekhi A, Shields CL. Photodynamic therapy for Extrafoveolar choroidal osteoma. Retina. 2020; 40(5):966-71. https://doi.org/10.1097//AE.0000000000002534.

11. Jabbehdari S, Fernández-Vega Gonzalez A, Zahid S, Mieler WF. Development of Choroidal Neovascularization after Treatment with Photodynamic Therapy in a 5-year old Female with Choroidal Osteoma. Retin Cases Brief Rep. 2020. https://doi.org/10.1097//CB.0000000000001089.

12. Shields CL, Materin MA, Mehta S, Foxman BT, Shields JA. Regression of extrafoveal choroidal osteoma following photodynamic therapy. Arch Ophthalmol. 2008;126(1):135-7. https://doi.org/10.1001/archopht.126.1.135.

13. Singh AD, Talbot JF, Rundle PA, Rennie IG. Choroidal neovascularization secondary to choroidal osteoma: successful treatment with photodynamic therapy. Eye (Lond). 2005;19(4):482-4. https://doi.org/10.1038/sj.eye.6701475.

14. Rose SJ, Burke JF, Brockhurst RJ. Argon laser photoablation of a choroidal osteoma. Retina. 1991;11(2):224-8. https://doi.org/10.1097/00006982-199111 020-00007.

15. Shukla D, Tanawade RG, Ramasamy K. Transpupillary thermotherapy for subfoveal choroidal neovascular membrane in choroidal osteoma. Eye (Lond). 2006;20(7):845-7. https://doi.org/10.1038/sj.eye.6702016.

16. Ahmadieh H, Vafi N. Dramatic response of choroidal neovascularization associated with choroidal osteoma to the intravitreal injection of bevacizumab (Avastin). Graefes Arch Clin Exp Ophthalmol. 2007;245(11): 1731-3. https://doi.org/10.1007/s00417-007-0636-z.

17. Navajas EV, Costa RA, Calucci D, Hammoudi DS, Simpson ER, Altomare F Multimodal fundus imaging in choroidal osteoma. Am J Ophthalmol. 2012; 153(5):890-5 e3. https://doi.org/10.1016/j.ajo.2011.10.025.

18. Cennamo G, Romano MR, lovino C, Velotti N, Breve MA, de Crecchio G, et al. OCT angiography in choroidal neovascularization secondary to choroidal osteoma. Acta Ophthalmol. 2017;95(2):e152-4. https://doi.org/1 $0.1111 /$ aos. 13142

\section{Publisher's Note}

Springer Nature remains neutral with regard to jurisdictional claims in published maps and institutional affiliations.
Ready to submit your research? Choose BMC and benefit from:

- fast, convenient online submission

- thorough peer review by experienced researchers in your field

- rapid publication on acceptance

- support for research data, including large and complex data types

- gold Open Access which fosters wider collaboration and increased citations

- maximum visibility for your research: over $100 \mathrm{M}$ website views per year

At $\mathrm{BMC}$, research is always in progress.

Learn more biomedcentral.com/submission 